\title{
Experimental Evaluation of Opportunistic Spectrum Access in Distributed Cognitive Radio Networks
}

\author{
Ahmed Khattab, Dmitri Perkins, Magdy A. Bayoumi \\ The Center for Advanced Computer Studies (CACS) \\ University of Louisiana at Lafayette \\ \{akk6481,perkins,mab\}@ cacs.louisiana.edu
}

\begin{abstract}
Opportunistic Spectrum Access (OSA) is foreseen as the future of wireless communications. However, today's cognitive radio technologies lag far behind the OSA goals and do not allow for the applicability of exiting theoretical distributed OSA techniques. In this paper, we experimentally demonstrate the ability of realizing OSA despite the practical limitations of existing transceiver technologies. We use the general purpose Wireless open-Access Research Platform (WARP) to instrument the implementation of fundamental OSA functionalities. Then we implement a suite of OSA schemes using this implementation framework. Our experiments show that suboptimal but practical OSA approaches such as random spectrum sensing and nongreedy access achieve superior performance given cognitive radios with limited capabilities compared to OSA approaches that are optimized for fully-capable cognitive radio networks (e.g., with wide-band sensing capability and adopt winner-takesall access relying on network-wide coordination mechanisms).

Index Terms-cognitive radio networks; opportunistic spectrum management; performance evaluation
\end{abstract}

\section{INTRODUCTION}

Opportunistic Spectrum Access (OSA) is a promising technique for tackling the spectrum scarcity problem by exploiting temporally unutilized spectrum bands [1], [2]. Opportunistic access schemes are the focus of significant research interest, especially from a theoretical perspective. The resulting theoretical approaches are challenged by the practical limitations of cognitive radios: the key enabling technology of OSA. Our focus is on the less well-studied issue of implementing distributed opportunistic spectrum access techniques given practical radio transceiver technologies and to characterize the gains provided by different OSA approaches in real systems.

In our prior work, we presented the Rate-Adaptive Probabilistic (RAP) OSA framework and protocol (RAP-MAC) that takes the practical limitations of existing cognitive radios into account while addressing the OSA problem [3]. More specifically, RAP targets distributed cognitive radio networks with limited hardware capabilities (e.g., narrow-band sensing) that rely only on local spectrum measurements to make spectrum access decisions based on sensing the activities of primary senders rather than the actual interference at primary receivers. Hence, such networks are susceptible to making wrong spectrum access decisions [1]-[3]. Furthermore, RAP

This work was supported in part by the National Science Foundation (NSF) under NSF Career Grant No. 0448055, the U.S. Department of Energy (DOE) under Award Number DE-FG02-04ER46136, and by the State of Louisiana, Louisiana Board of Regents under Contract Numbers DOE/LEQSF(2004-07)ULL and LEQSF(2003-06)-RD-A-35. does not allow for explicit inter-flow coordination to avoid the associated overhead and other challenges resulting from the use of a common control channel for global network coordination. ${ }^{1}$ The RAP OSA approach uses coordinated random spectrum selection combined with a rate-adaptive probabilistic access mechanism to counter the unavoidable inaccuracies of spectrum management in such low-complexity distributed cognitive radio networks while achieving fair spectrum sharing. Using analysis and packet-level simulations in arbitrary largescale networks, RAP OSA was shown to achieve up to $140 \%$ goodput gain with remarkable fairness performance compared to the hypothetically-optimal approaches that assume currently unavailable transceivers and explicit inter-flow coordination.

In this paper, we use the Wireless open-Access Research Platform (WARP) [4] not only to demonstrate the superior performance of the RAP approach in a real system but also to thoroughly investigate the role of the individual RAP components in the overall performance and their applicability to other existing OSA approaches. WARP is well recognized by both the academic and industrial research communities for cleanslate prototyping. First, we instrument the basic functions common to different OSA approaches. Then we implement the RAP approach as well as a set of other OSA approaches needed for our performance evaluation study. Despite using randomized and non-greedy spectrum access decisions, our results show that the RAP approach achieves significantly higher goodput (from $66 \%$ and up to multiple folds gain depending on the primary networks' activities) compared to other OSA techniques that use greedy access approaches based on wide-band sensing. Random sensing contributes to $70 \%$ to $80 \%$ of this gain, while the remaining $20 \%$ to $30 \%$ gain is due to the probabilistic access mechanism. This superior goodput performance comes at the expense of higher primary networks' outages due to the random and probabilistic decisions. However, the primary network outages do not violate the targeted bounds. Furthermore, our experiments demonstrate that existing theoretical OSA approaches can benefit the gains of either randomized sensing or non-greedy probabilistic access.

The remainder of the paper is organized as follows. In Section II, we describe our hardware implementation framework. Then, we briefly present the OSA protocols we use for our performance evaluation in Section III. In Section IV,

${ }^{1}$ However, RAP still uses a control channel to coordinate the spectrum decision between a cognitive sender and its receiver. 
we present an extensive set of experiments to evaluate the performance of different OSA approaches. We overview the related work in Section V and conclude in Section VI.

\section{Opportunistic Spectrum ACCESS Implementation FRAMEWORK}

The Wireless open-Access Research Platform (WARP) is an FPGA-based hardware platform with an open-source repository of wireless building blocks and reference designs [4]. The WARP implements an OFDM transceiver on the fabric of the FPGA. WARP is ideal for clean-slate medium access prototyping through a flexible interface between the physical and medium access layers. ${ }^{2}$ Using the WARP OFDM physical layer, we develop a framework for implementing OSA protocols. The OSA implementation framework is written in C-langauge, compiled and downloaded to one of the PowerPC cores of a WARP board where it directly interacts with the physical layer implementation.

Our implementation framework instruments the basic functionalities commonly used by different distributed opportunistic spectrum management schemes. We implement the following four mechanisms using the WARP OFDM reference design version 14: (i) spectrum sensing, (ii) common control channel, (iii) spectrum coordination packet handshake, and (iv) multi-rate multi-power packet transmission.

- Spectrum Sensing. The purpose of this function is to measure the cumulative interference of a given spectrum band and determine whether it is below the power mask specified by the corresponding primary network or not. This is realized by monitoring the received signal strength indicator (RSSI) averaged over a certain time window. By comparing the time-averaged RSSI with the spectrum power mask, an opportunistic spectrum access protocol can determine whether this band is clear (RSSI $<$ Power Mask) or not (RSSI $\geq$ Power Mask).

- Common Control Channel. Distributed opportunistic spectrum access protocols require a means by which a cognitive sender coordinates its spectrum decisions with its intended receiver. A common control channel is generally used for this purpose. Both the sender and the receiver are continuously listening to this channel if not involved in an active data exchange. We define channel 14 of the $2.4 \mathrm{GHz}$ ISM band as the common control channel. Channel 14 of the $2.4 \mathrm{GHz}$ band is not available for commercial purposes in the United States and can only be used for academic research. Using such a channel guarantees a robust common control channel.

- Spectrum Coordination Packet Handshake. We create the control packets to be exchanged over the common control channel for cognitive sender-receiver coordination. These control packets do not include any payload bytes and only include the sender and the intended receiver addresses in addition to other protocol-dependent control information such as the selected spectrum, the

\footnotetext{
${ }^{2}$ While software-defined radios provide a more flexible development environment, the throughput and latency of such platforms lag far behind the requirements of real-life communications systems despite their low cost [5].
}

measured RSSI, the modulation rate, etc. For RAP and the other tested OSA protocols, we only need a twoway control-message handshake in which the sender informs its receiver with its spectrum selections via a control packet and the receiver confirms or denies such selections with another control packet. The control packet handshake is transmitted using the base rate realized via the WARP QPSK modulation scheme.

- Multi-rate Multi-power Packet Transmission. Finally, we implement a data packet transmission scheme which parameters are configured on a packet-per-packet basis. For the considered opportunistic spectrum management schemes, we allow the protocol to configure the transmission channel, the modulation rate and power. A data packet can use one out of three WARP modulation schemes: BPSK, QPSK, and 16 QAM with respective transmission powers of $12 \mathrm{dBm}, 15 \mathrm{dBm}$, and $18 \mathrm{dBm}$.

\section{PRotocol IMPLEMENTATIONS}

Our objective is not only to demonstrate the advantages of the RAP OSA approach but also to study how much gain is attributed to its different practical mechanisms. Moreover, we also show how traditional OSA approaches can benefit from the individual RAP components. Consequently, we implement the following suite of OSA protocols needed for our empirical performance evaluation.

\section{A. Random Sensing with Probabilistic Access}

The first OSA protocol we implement is the RAP-MAC protocol that we developed in [3] for low-complexity and practical cognitive radio networks. To counter the lack of a mechanism to assess the interference at the primary receivers and to avoid the overhead of explicit inter-flow coordination, RAP-MAC adopts coordinated random spectrum selection combined with a rate-adaptive probabilistic transmission policy. The coordinated random spectrum selection component has the sender randomly selecting a spectrum followed by a two-way message handshake to exchange the assessment of the interference over that spectrum at both the sender and the receiver. Hence, both the flow's endpoints participate in the decision of weather or not to use the randomly-selected spectrum. The random sensing component relaxes the requirements of the sensing module of a cognitive radio (since it does not require a wideband front-end).

Meanwhile, the rate-adaptive probabilistic component has the sender probabilistically deciding whether or not to transmit and at which rate and power based on the two interference measurements at both the flow's endpoints and the fact that these measurements do not actually reflect the interference at nearby primary receivers. The RAP-MAC access policy is nongreedy in the sense that the highest rate and power applicable to a given scenario are not used with a unity probability. Instead, lower rates and powers are to be probabilistically used to $(i)$ counter the unavoidable inaccuracy in spectrum sensing due to hidden and exposed primary nodes (since spectrum sensing techniques only measure the transmission activities of the primary senders), and (ii) prevent a single cognitive senderreceiver pair from monopolizing a spectral opportunity to 
allow multiple flows to share such an opportunity, and hence, alleviates the need for explicit inter-flow coordination. Further details of the RAP-MAC framework and protocol details are available in [3]. We refer to such an OSA approach as random sensing with probabilistic access.

\section{B. Sequential Sensing with Greedy Access}

This implementation reflects the wide range of existing opportunistic spectrum access protocols (e.g., [6], [7]). In such schemes, a cognitive radio node senses all of the available spectrum bands before deciding which band to use. Unlike the RAP-MAC approach, such schemes adopt deterministic and greedy (i.e., winner-takes-all) access mechanisms in which a sender only transmits if there exists a spectrum which its measured RSSI is below the power mask. Furthermore, such senders transmit using the highest possible power and rate for all the time. We use a modified version of a candidate protocol of this family of protocols that was presented in [7] for our implementation. The spectrum access mechanism of such protocols is based on traditional carrier sensing that uses a two-way message exchange over the common control channel to insure a single secondary user transmission per contention area. However, we do not implement the ability of sensing or transmitting over multiple bands simultaneously as in the original protocol presented in [7] to adapt to the limited capabilities of conventional transceivers and for the sake of fairness in comparison. Recall that the WARP transceiver can be tuned to only one frequency channel at a time as the case with other contemporary single-radio transceivers. Therefore, we implement a sequential spectrum sensing mechanism in which a cognitive node goes over the channels of interest and reports back the RSSI of individual channels.

\section{Sequential Sensing with Probabilistic Access}

The second protocol that we use for comparison is a derivative of the above implementation which still depends on sequentially scanning all of the available spectrum bands before deciding the best spectrum to use based on traditional carrier sensing. However, this protocol adopts a probabilistic and nongreedy spectrum access approach similar to that developed for the RAP-MAC protocol instead of using deterministic and greedy spectrum access. Such a protocol helps identifying how much gain can be achieved by using a probabilistic access mechanism if adopted by the wide range of existing protocols that rely on greedy access strategies. Furthermore, this protocol implementation allows us to assess how much gain of the RAP approach is due to random sensing since the sensing mechanism is the only difference between RAP-MAC and this protocol implementation.

\section{Random Sensing with Greedy Access}

We also implement a variant of the RAP-MAC protocol in which RAP probabilistic access is replaced with traditional winner-takes-all access. We refer to this protocol implementation as the random sensing with greedy access protocol. The greedy access mechanism of this protocol is the same one used by the sequential sensing with greedy access protocol. Hence, this protocol allows us to quantify the performance gain of randomized narrow-band sensing compared to sequential wideband sensing. Furthermore, comparing the performance of this protocol implementation against the RAP-MAC illustrates the contribution of the RAP probabilistic access component in the overall RAP gain as will be demonstrated by our experiments.

\section{Performance Evaluation}

\section{A. Experimental Setup}

Implementing a cognitive radio network (CRN) environment poses significant design challenges. For example, any opportunistic spectrum access experiment requires the creation of multiple primary networks (PRNs) which spectral opportunities can be exploited by the cognitive radio network users when the primary users are inactive. Thus, the experiments must provide controllable primary network flows. Furthermore, the experimental setup must keep track of every cognitive radio network transmission as well as every primary networks' transmission and reception in order to assess the CRN decision mechanism and the outage performance of the primary networks, respectively.

Primary Networks Implementation. For our experiments, we create two primary networks each composed of a single sender and a single receiver. In order to have full control over the primary networks' performance and to not harm existing licensed networks, we configure the two primary networks to operate over non-overlapping channels in the unlicensed 2.4 $\mathrm{GHz}$ ISM band. More specifically, we configure the first PRN to use channel 1 of the $2.4 \mathrm{GHz}$ and the second PRN to use channel 7 of the same band. We use laptops equipped with IEEE 802.11 wireless cards to create the primary networks. The transmission power of each network is set to $18 \mathrm{dBm}$ and the physical layer transmission rate is set to $11 \mathrm{Mbps}$ with the auto-rate feature turned off. We use iperf to generate a UDP flow from each primary sender and collect the UDP flow statistics at the corresponding receiver. We measure the backlog UDP capacity of the two primary network in the absence of any cognitive radio network activities to be 6.03 Mbps and 6.15 Mbps, respectively.

Cognitive Radio Network Implementation. We create a cognitive radio node by connecting a laptop (with its wireless interface disabled) to a WARP board via the WARP Ethernet port. By downloading the appropriate bit file of any of the implemented opportunistic spectrum access protocols to a WARP PowerPC, the WARP board will act as the wireless air interface of the laptop that runs that particular OSA protocol. We create a fully backlogged cognitive radio transmission between two such cognitive radio nodes using iperf. The cognitive radio sender and receiver nodes are at equal distance of approximately 2 meters from the senders and receivers of the two collocated primary networks. Table I summarizes the experiment parameters and Figure 1 depicts a layout of the experiment setup. ${ }^{3}$

\footnotetext{
${ }^{3}$ While RAP was shown to have remarkable fairness in distributing the spectral opportunities among multiple competing cognitive flows, we canno experimentally demonstrate such a performance aspect due to the associated cost and complexity. We refer interested readers to [3] for a thorough performance evaluation of large-scale networks using simulations.
} 


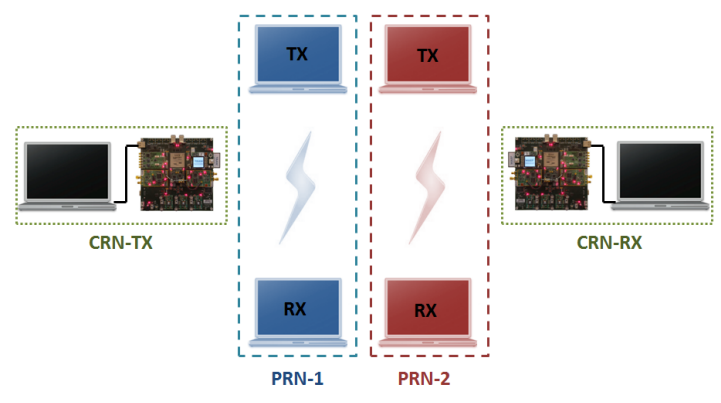

Fig. 1. Illustration of the experiment setup.

TABLE I

EXPERIMENT PARAMETERS SUMMARY.

\begin{tabular}{|c|c|}
\hline Parameter & Value \\
\hline \hline Channel switching time & $10 \mu \mathrm{sec}$ \\
\hline Timeout period & $50 \mu \mathrm{sec}$ \\
\hline Sensing time per channel & $30 \mu \mathrm{sec}$ \\
\hline Transceiver turnaround time & $23 \mu \mathrm{sec}$ \\
\hline Payload packet length & $1450 \mathrm{Bytes}$ \\
\hline Control packet length & $24 \mathrm{Bytes}$ \\
\hline TX power (BPSK, QPSK, 16QAM) & $(12,15,18) \mathrm{dB}$ \\
\hline MAC data rate (BPSK, QPSK, 16QAM) & $(4.1,8.410 .4) \mathrm{Mbps}$ \\
\hline
\end{tabular}

Our performance metrics are both the goodput of the cognitive radio flow (defined as the amount of data correctly received at the receiver) as well as the outage probability of both primary networks (defined as the percentage of the loss in the transmission rate due to the activity of the cognitive radio transmission). The reported results in the next subsection are the average of several runs each of one minute length. We run the experiments between midnight at the early hours of the morning to minimize the potential uncontrolled transmission activities over the used channels. A demonstration of the RAPMAC implementation in action is available online at [8].

\section{B. Experimental Results}

1) Baseline Experiment: We start by characterizing the performance of the RAP approach in the worst-case scenario in which the primary networks are fully utilized. Our goal is to identify the values of the parameters of the RAP rate-adaptive probabilistic component. Note that the optimal parameter values analytically derived in [3] for arbitrary large-scale networks do not directly apply to our testbed setup due to the difference in the underlying system assumptions. We perform a two-dimensional sweep of the probability of transmission when the spectrum is clear and unclear. For a targeted 5\% maximum primary networks' outage, the measured values of the transmission probabilities were 0.4 and 0.4 in the clear and unclear spectrum scenarios, respectively. We use these values for the rest of our experiments.

2) CRN Goodput Performance: Figure 2 illustrates the goodput achieved by the cognitive radio flow according to different protocol implementations. We vary the activity factor of both primary networks by varying the UDP flow rate such that the primary networks' activities go from idle to fully backlogged in $25 \%$ activity increments. As shown in Figure 2(a),

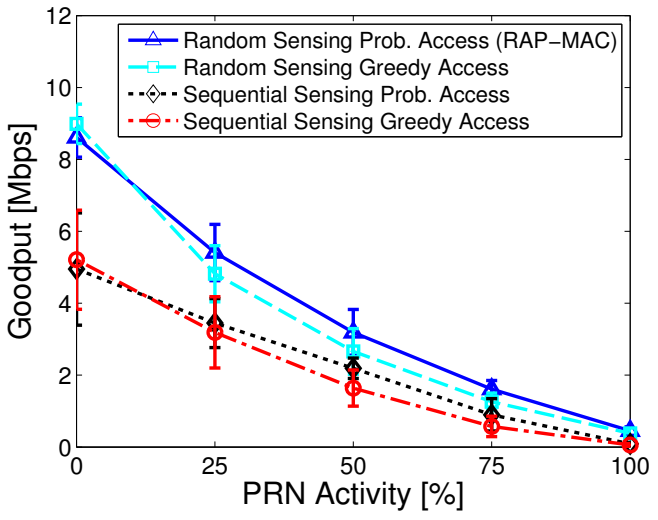

(a) CRN flow goodput.

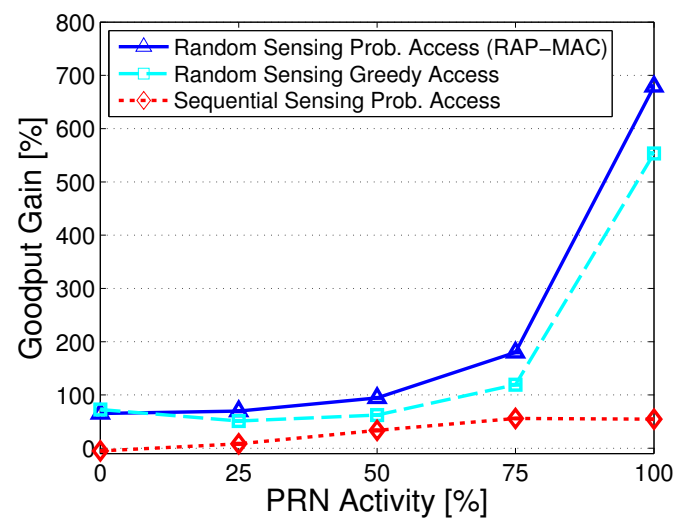

(b) Gain w.r.t. sequential sensing with greedy access.

Fig. 2. RAP-MAC achieves significant goodput gain over traditional opportunistic spectrum access schemes. While both components contribute to the overall gain, the goodput gain due to randomized sensing is higher than the gain due the probabilistic access mechanism.

RAP-MAC achieves the highest cognitive flow goodput when the PRNs are not idle, while the sequential sensing with greedy access approach widely used for opportunistic spectrum access results in the lowest goodput. The RAP-MAC goodput gain increases from $66 \%$ at low primary networks' activities to $95 \%$ at $50 \%$ PRN activity as shown in Figure 2(b). As the PRN activities increase, the RAP-MAC goodput becomes multiple folds of the goodput achieved by the benchmark protocol until the RAP-MAC goodput is 6.7 times the benchmark goodput when the PRNs are fully backlogged.

The superior goodput performance of RAP-MAC is attributed to both its main components: the randomized sensing component (which alleviates the overhead of scanning all frequencies before a given access by measuring the interference on only one randomly-selected frequency) and the nongreedy probabilistic access component (which probabilistically explores the spectral opportunities rather than adopting the traditional winner-takes-all approach). We use the goodput achieved by the other two protocol implementations (shown in Figure 2(a)) to perform pairwise comparisons to identify how much each component is contributing to the overall gain. Intuitively, adopting a greedy access strategy results in slightly 
higher goodput when the primary networks are idle, regardless of the sensing mechanism. Hence, random sensing is the main contributor to the overall gain at low PRN activities as seen by comparing the protocols implementing random sensing against sequential sensing for both access mechanisms. More specifically, by comparing the solid blue curve (representing RAP-MAC) against the black dotted curve (representing its sequential sensing counterpart), and comparing the dashed light blue curve (representing random sensing with probabilistic access) against the dash-dotted red curve (representing the common greedy access based on sequential sensing). As the activities of the primary networks increase, the gain due to probabilistic access increases. For primary networks' activities of $50 \%$ and above, the contribution of random sensing is approximately $70 \%$ to $80 \%$ of the overall RAP-MAC gain while the contribution of the probabilistic access mechanism is around $20 \%$ to $30 \%$ depending on the PRN activities.

Furthermore, the individual components of RAP-MAC can be used to improve the performance of the family of spectrum management approaches that uses sequential sensing with greedy access as shown in Figure 2(b). For example, adopting a non-greedy access mechanism can result in goodput gain of up to $56 \%$ as depicted by the dotted red curve. Furthermore, exploiting random sensing instead of sequentially searching for the best channel to use achieves $64 \%$ to $82 \%$ of the RAPMAC gain, depending on the PRN activities, as illustrated by the light-blue dashed curve. This emphasizes that the random sensing component has a more significant performance gain.

3) PRN Outage Performance: Next, we evaluate the outage performance of the primary networks for the different opportunistic spectrum access protocol implementations. Figure 3 depicts the outage probabilities of both primary networks for all protocol implementations versus the activity of the primary networks. Two observations can be made regarding Figure 3. First, probabilistic access schemes result in slightly higher PRN outages compared to their greedy access counterparts. However, probabilistic access has a weaker impact on the PRN outage when sequential sensing is used (as illustrated by the small gap between the dotted black and dash-dotted red outage curves). With the inaccuracies of random sensing, the impact of probabilistic access increases (as illustrated by the gap between the solid blue and dashed light-blue outage curves). Second, random sensing results in approximately 2.6 times the outages due to sequential sensing protocol irrespective of the access protocol. This is because sequential sensing protocols assess the interference levels on both channels before deciding the transmission action. On the other hand, random sensing protocols simply pick a channel at random for transmission. Note that despite resulting in higher primary network outages, random sensing protocols including RAP-MAC adhere to the targeted 5\% maximum outage constraint. However, the significant multi-fold goodput gain of such protocol illustrated in Figure 2 outweighes the excess primary outages resulting from such protocols. Furthermore, as the number of the primary networks increases, the sensing time required to assess the interference on all channels will increase. Hence, the RAP goodput gain is expected to further increase.

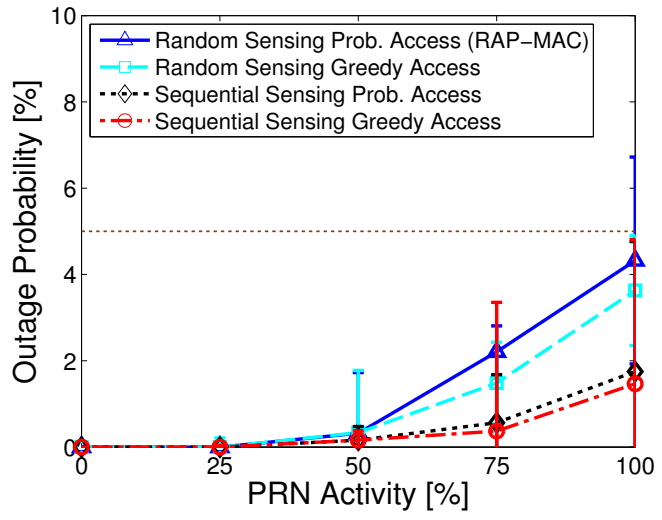

(a) Primary network operating using channel 1.

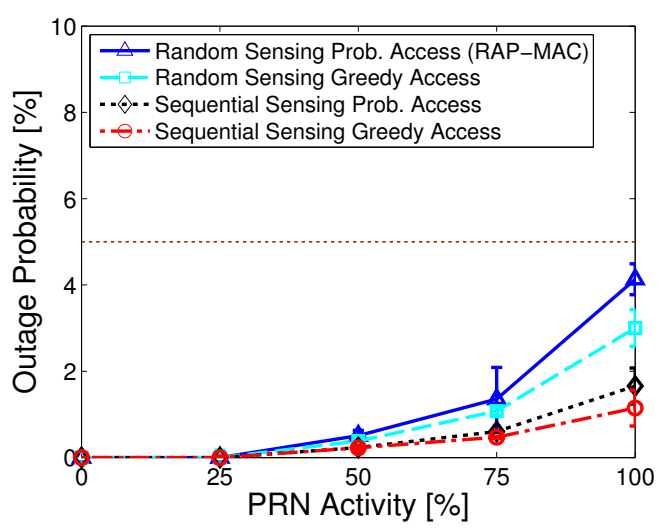

(b) Primary network operating using channel 7.

Fig. 3. The outage probability of the primary networks versus the activity factor for different protocol implementations. While both satisfy the 5\% PRN outage constraints, random sensing results in more primary outages compared to sequential sensing.

4) Channel Utilization: Finally, we examine the channel utilization of different OSA approaches. While randomsensing-based OSA protocols randomly pick the used spectrum, they tend to have spectrum utilization patterns similar to those OSA schemes that sense the entire spectrum. Figure 4 depicts the percentage each channel is utilized for RAP-MAC and the sequential sensing with greedy access protocol. At low primary networks' activities, both approaches tend to evenly exploit both channels. As the activities of the primary networks increase, both protocols tend to use channel 7 more often than channel 1 . However, the gap in the utilization percentage is smaller and only appears at very high primary network activities for the sequential sensing with greedy access due to its informed spectrum selection mechanism.

\section{RELATED WORK}

Existing hardware/software platforms that can be used to implement opportunistic spectrum access protocols can be classified into two main classes: Software Defined Radio (SDR)-based and Field Programmable Gate Array (FPGA)based platforms. SDR platforms are implemented via the integration of the GNU Radio that is a software development 


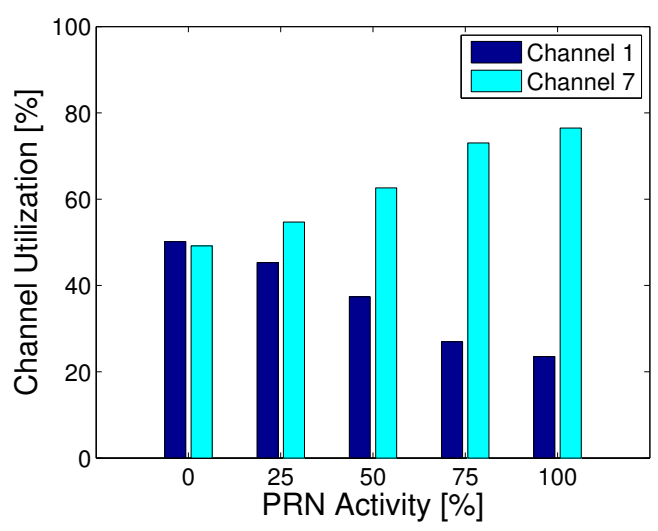

(a) Random sensing with probabilistic access (RAP-MAC).

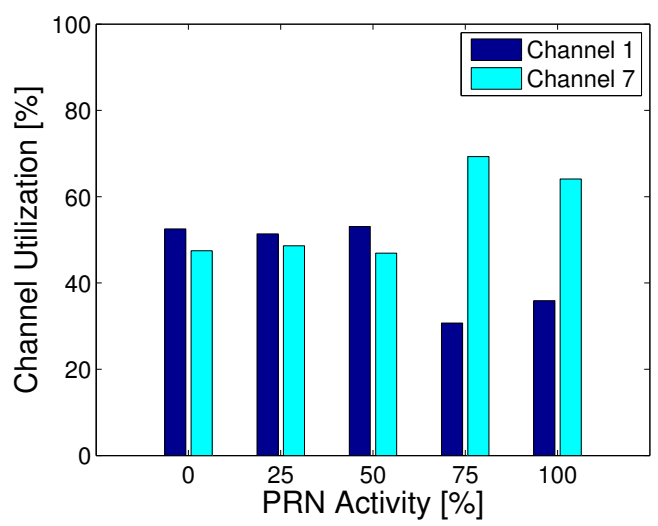

(b) Sequential sensing with greedy access.

Fig. 4. While both approaches tend to exploit channel 7 more often, the informed and deterministic spectrum management actions of the sequential sensing with greedy access protocol lead to a more balanced utilization pattern.

environment [9] and any of the Universal Software Radio Peripheral (USRP) product family that is used as the RF interface of the platform [10]. SDR platforms provide more flexibility in implementing spectrum sensing and spectrum management since they rely on software to implement such functionalities. Different open-source GNU radio software specifically written for cognitive radio networks are available such as the Cognitive Radio Open Source System (CROSS) [11], and the Papyrus software platform [12]. However, the throughput and latency of the prototypes implemented via SDR platforms are one to three orders of magnitude worse than realistic hardware designs and lag far behind the requirements of real-world communication schemes such as IEEE 802.11 [5] - despite their low cost.

On the other hand, FPGA-based platforms offer orders of magnitude improvement in the latency and throughput performance at the expense of increased hardware complexity and cost. An FPGA-based platform is often composed of a hardware component that consists of a compact FPGA board which implements the physical and link layers associated with a software environment that provides the basic physical and MAC layers functionalities and interfaces to the hardware component that allow the researchers to program the hardware as desired. Thus, FPGA-based systems combine the programmability of software and the high performance and predictability of hardware. Although other platforms exists (e.g., AirBlue [5]), we have chosen the Wireless open-Access Research Platform (WARP) [4] FPGA-based platform for our empirical performance evaluation study. A prototype of OSA implementation using WARP was presented in [13]. However, that prototype is a derivative of the IEEE 802.11 medium access approach. In contrast, we present the first implementation and performance evaluation of clean-slate opportunistic spectrum access approaches.

\section{CONCLUSions}

In this paper, we have presented an experimental study of the less-well studied topic of distributed opportunistic spectrum access implementation. Our goal is to demonstrate that while existing hardware technologies do not provide the cognitive transceiver requirements needed to exploit OSA to its full potential, suboptimal OSA approaches developed to target low-complexity transceivers can achieve significant performance improvement compared to theoretically-optimal approaches. More specifically, we have shown that the use of random spectrum selection combined with non-greedy and probabilistic access leads to up to multiple folds goodput gain at the expense of higher primary outage (within the permissible bounds). We have also shown that other theoretical OSA approaches can exploit the gains of either techniques. We plan to further extend our experiments to consider more primary networks and multi-flow cognitive networking to study the fairness performance of different approaches.

\section{REFERENCES}

[1] I. F. Akyildiz, W.-Y. Lee, and K. R. Chowdhury, "CRAHNs: cognitive radio ad hoc networks," Ad Hoc Networks (Elsevier), vol. 7, no. 5, pp. 810-836, Jul. 2009.

[2] H. B. Salameh and M. Krunz, "Channel access protocols for multihop opportunistic networks: challenges and recent developments," IEEE Networks, vol. 23, no. 4, pp. 14-19, Jul. 2009.

[3] A. Khattab, D. Perkins, and M. A. Bayoumi, "Rate-adaptive probabilistic spectrum management for cognitive radio networks," in Proc. of the IEEE WoWMoM 2011, Lucca, Italy, Jun. 2011.

[4] "Rice University WARP Project." [Online]. Available: http://warp.rice.edu

[5] M. C. Ng, K. E. Fleming, M. Vutukuru, S. Gross, Arvind, and H. Balakrishnan, "Airblue: a system for cross-layer wireless protocol development," in Proc. of the 6th ACM/IEEE ANCS '10, Oct. 2010.

[6] J. Jia, Q. Zhang, and X. Shen, "HC-MAC: A hardware-constrained cognitive MAC for efficient spectrum management," IEEE Journal on Selected Areas in Commun., vol. 26, no. 1, pp. 106 -117, Jan. 2008.

[7] H. B. Salameh, M. Krunz, , and O. Younis, "MAC protocol for opportunistic cognitive radio networks with soft guarantees," IEEE Transactions on Mobile Computing, vol. 8, no. 10, pp. 1339-1352, Oct. 2009.

[8] "RAP-MAC demo." [Online]. Available: http://www.youtube.com/watch?v=8X-cbQFrLxM

[9] "GNU Radio." [Online]. Available: http://gnuradio.org/trac

[10] "Ettus Research LLC.” [Online]. Available: http://www.ettus.com/

[11] "CROSS: Cognitive Radio Open Source System." [Online]. Available: http://cornet.wireless.vt.edu/trac/wiki/Cross

[12] L. Yang, Z. Zhang, W. Hou, B. Y. Zhao, and H. Zheng, "Papyrus: a software platform for distributed dynamic spectrum sharing using SDRs," ACM SIGCOMM Comput. Commun. Rev., vol. 41, pp. 31-37, Jan. 2011.

[13] J. Ansari, X. Zhang, A. Achtzehn, M. Petrova, and P. Mahonen, "A flexible mac development framework for cognitive radio systems," in Wireless Communications and Networking Conference (WCNC), 2011 IEEE, Quintana Roo, Mexico, Mar. 2011. 